\title{
Linear and non-linear optical properties of GaAs nanowires
}

\author{
Satyendra Singh $\cdot$ Pankaj Srivastava
}

Received: 11 March 2014/ Accepted: 28 April 2014/Published online: 20 May 2014

(C) The Author(s) 2014. This article is published with open access at Springerlink.com

\begin{abstract}
The linear and non-linear optical properties of different geometrical structures of gallium arsenide (GaAs) nanowires have been studied by employing ab initio method. We have calculated the optical response of four different GaAs nanowires, viz., two-atom linear wire, twoatom zigzag wire, four-atom square wire and six-atom hexagonal wire. We have investigated imaginary part of the $z z$ component of the linear dielectric tensor and secondorder susceptibility for different structures along with bulk material. We revealed that the strongest absorption occurs for four-atom square nanowire configuration.
\end{abstract}

Keywords GaAs nanowires - Linear optical properties . Non-linear optical properties

\section{Introduction}

Gallium arsenide (GaAs) semiconducting nanowires with diameters ranging from 1 to $400 \mathrm{~nm}$ and length of up to hundreds of micrometers have shown remarkable optical properties and hence they will prove to be the versatile building blocks for optoelectronic circuits at a nanoscale. GaAs semiconducting nanowires also offer a unique approach for the bottom-up assembly of electronic and photonic devices with a potential for on-chip integration of

\footnotetext{
S. Singh $(\square)$

Department of Physics, Shri Ram College of Engineering and Management, Banmore, Morena 476444, Madhya Pradesh, India e-mail: satyendra7171@yahoo.co.in

\section{P. Srivastava}

Nanomaterials Research Group, Indian Institute of Information Technology and Management (ABV-IIITM), Gwalior 474010, Madhya Pradesh, India
}

non-silicon-based photonic devices with silicon nanoelectronics-based devices (Lieber 2001, 2003; Law et al. 2004; Xia et al. 2003; Dekker 1999; Collins and Avouris 2000). GaAs nanowires are also very promising candidates for the development of a number of new nanoscale optical devices a few to be named as nanowire-based photodetectors, single nanowire laser, etc. (Duan et al. 2000, 2001).

The existence of the exciton has a strong influence on the electronic properties of the semiconductor and its optical absorption. The second-order response or non-linear property is a two-photon process where the excited electron absorbs another photon of the same frequency and makes a transition to another allowed state at higher energy. When this electron is falling back to its original state, it emits a photon of frequency, which is twice the frequency of that of the incident light (Srivastava et al. 2008; Srivastava and Singh 2008; Singh and Srivastava 2013).

Electronic and optoelectronic applications demand straight, uniformly aligned nanowires with uniform diameters and excellent crystallographic, electronic and optical properties; currently, the usefulness of GaAs nanowires in these devices is limited due to the presence of certain defects like crystallographic twin defects (Ihn and Song 2007), nanowire kinking (Zou et al. 2007), intrinsic doping and surface states (Joyce et al. 2007). Imaging and optical properties of single core-shell GaAs-AlGaAs nanowires were studied by Hoang et al. (Titova et al. 2006). Duan et al. (2000) synthesized and studied the optical properties of gallium arsenide nanowires. Electronic and optical properties of InAs/GaAs nanowires were studied by Niquet (2006). Redliski and Peters (2008) studied the optical properties of free-standing GaAs semiconducting nanowires and their dependence on the growth direction. Growth, structural and optical properties of GaAs/AlGaAs nanowires with and without quantum well shells were 


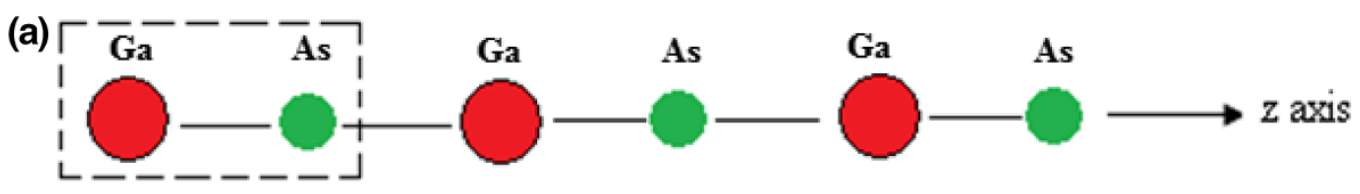

two-atom linear wire

(b)

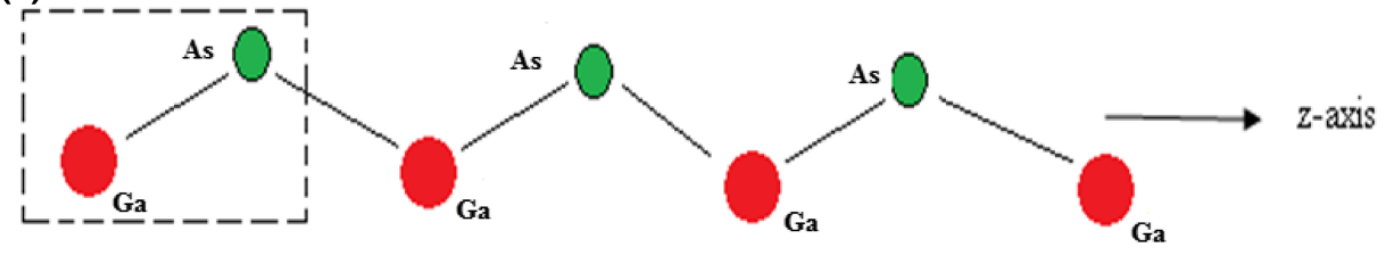

two-atom zigzag wire

(c)

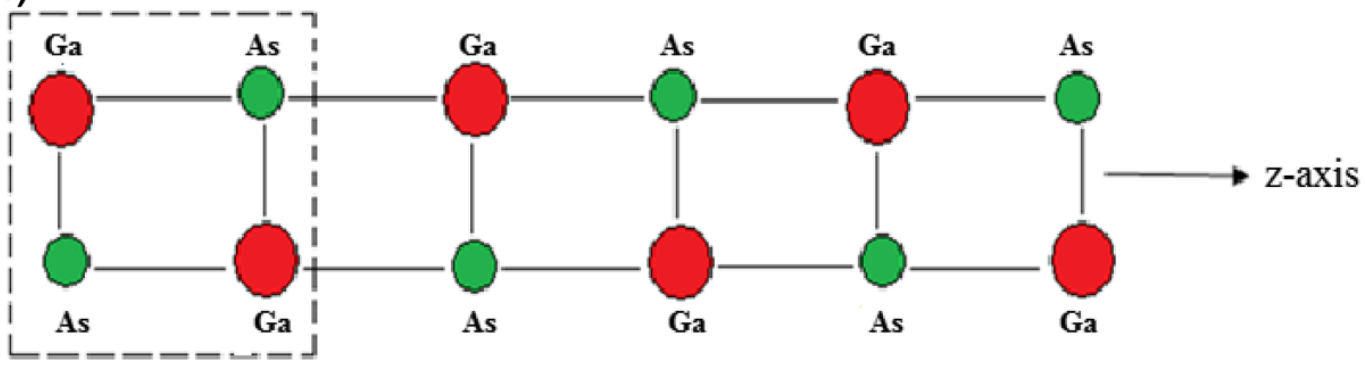

four-atom square wire

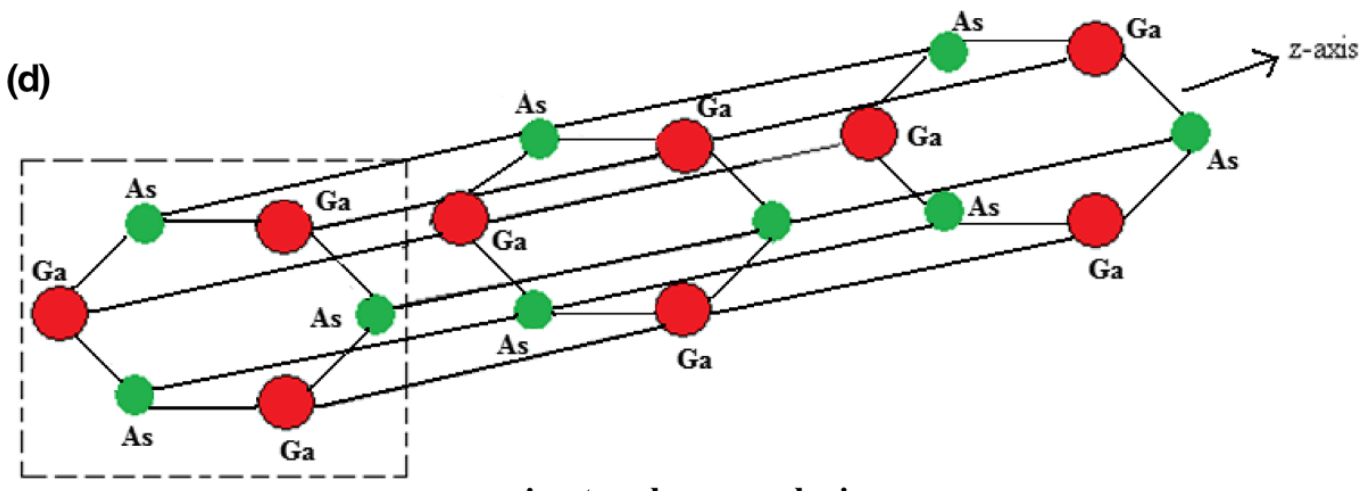

six-atom hexagonal wire

Fig. 1 Optimized structures of GaAs nanowires: a two-atom linear wire, $\mathbf{b}$ two-atom zigzag wire, $\mathbf{c}$ four-atom square wire, $\mathbf{d}$ six-atom hexagonal wire

demonstrated by Joyce et al. (2006). GaAs nanowires for optoelectronics were studied by Kim et al. (2006). Hoang et al. (2009) studied the optical properties of single wurtzite GaAs nanowires and GaAs nanowires with GaAsSb inserts. Zhang et al. (2009) synthesized the GaAs nanowires with very small diameters and studied their optical properties with the radial quantum-confinement effect. The study of effect of structure, surface passivation and doping on the electronic and optical properties of GaAs nanowires by a first principle study was done by Khare et al. (2009).

Four different geometrical structures of GaAs nanowires are considered and the linear and non-linear optical properties of considered structures are being presented in this paper. The linear and non-linear spectra for considered structures are investigated and finally the optical transition in different energy regions is discussed. The details about the geometrical structures have been given in our earlier 
published papers (Srivastava et al. 2008; Singh et al. 2009a, b; Srivastava and Singh 2008, 2011; Srivastava et al. 2011). The considered geometrical structures of GaAs nanowires are shown in Fig. 1.

\section{Methodology}

ABINIT code (Hohonberg and Kohn 1964; Kohn and Sham 1965) has been used for the computational work. The ab initio DFT calculations (Martin 2009; Gonze et al. 2002) are employed within the plane-wave pseudopotential method to investigate the linear and non-linear optical properties of $\mathrm{GaAs}$ nanowires. As is evident from the above literature that the pseudopotential method has been very successful in exploring the structural, electronic and optical properties of various materials (Martin 2009); in this calculation, the generalized gradient approximation and the exchange-correlation functional of Perdew et al. (1996) were applied. The exchange correlation potential of Troullier and Martins (1991) has been used and these pseudopotentials were taken from ABINIT web page (Gonze et al. 2002). The potentials were tested by performing calculations on bulk GaAs material in which the results were found to be consistent with the experimental ones. All the calculations were performed in a self-consistent manner. The studied structures were optimized for Hellmann-Feynman forces as small as $10^{-3} \mathrm{eV} / \mathrm{A}$ on each atom and the calculations were performed with a kinetic energy cut-off of $816.3418 \mathrm{eV}$. The wires were positioned in a supercell of side $10.583 \AA$ along the $x$ and $y$ directions; the axis of the wire was taken along the $z$ direction and the periodic boundary conditions were applied. The Monkhorst-Pack method with $15 \mathrm{k}$-points sampling along the $z$ direction was used in the integration of the Brillouin zone; all atoms were allowed to relax without any imposed constraint. In order to check the self-consistent calculations, we have determined the self-consistent optimized value for the lattice parameter of bulk GaAs materials, the magnitude of atomic relaxation depends on the plane cutoff energy and one has to obtain the convergence with respect to cut-off energy too.

\section{Results and discussion}

The imaginary parts of the $z z$ component of the linear dielectric tensor or linear susceptibility for various gallium arsenide (GaAs) nanowires along with bulk are presented in Fig. 2. The linear optical response of bulk is quite smooth and the linear susceptibility gradually increases with energy, for two-atom linear wire, it is observed that there are two strong peaks around 4.5 and $5.8 \mathrm{eV}$ with

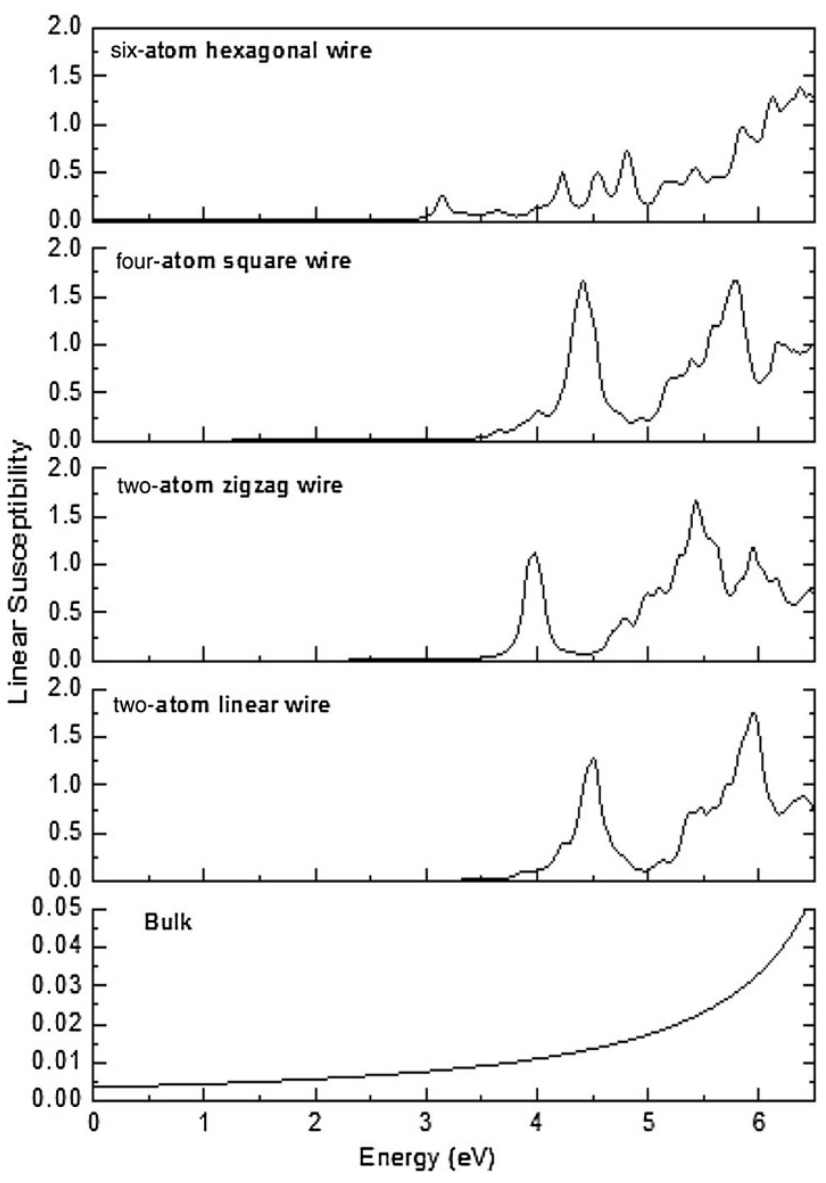

Fig. 2 Calculated imaginary part of the $z z$ component of the linear dielectric tensor for different GaAs nanowires along with bulk GaAs material

small peaks towards higher energy side, but in the case of a two-atom zigzag wire, strong absorption is observed with three major peaks at 4.0, 5.5 and $6.0 \mathrm{eV}$. Beside this there are other peaks also present. The four-atom square wire linear spectra has strongest peak around 4.2 and $5.8 \mathrm{eV}$, which exhibit strong absorption in such type of geometrical configuration. The other weak absorption peaks at higher energy region are also seen. For a six-atom hexagonal wire cross-section, peaks occur in between 4.0 and $6.5 \mathrm{eV}$.

Thus, from linear absorption spectra of all the configurations, it is predicted that the strongest absorption is shown by four-atom square wire. In the study of electronic properties, it can be presented that same structure having the highest stability (Singh et al. 2009a, b), thus it can be predicted that the same structure that has the highest stability, must also have the strongest absorption which is evident from our investigation.

The non-linear optical spectra of different configuration of GaAs nanowires are analyzed and various contributions to the imaginary part of $\chi_{z z z}(2 \omega, \omega, \omega)$ are presented in Figs. 3, 4, 5, 6, 7. 
Fig. 3 Calculated second-order susceptibility $I_{m}\left[\chi_{z z z}(2 \omega, \omega\right.$, $\omega)]$ and different contributions for the bulk GaAs material

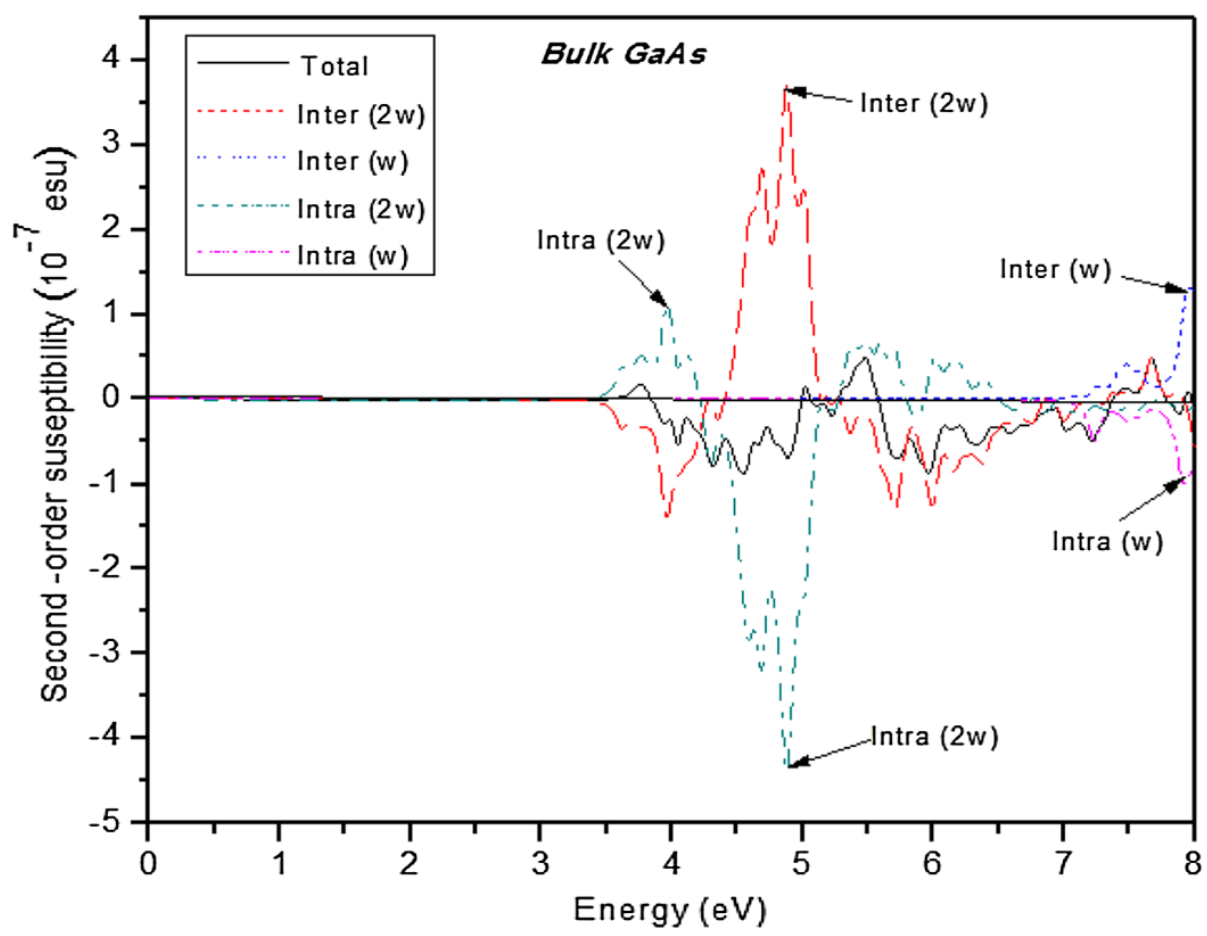

Fig. 4 Calculated second-order susceptibility $I_{m}\left[\chi_{z z z}(2 \omega, \omega\right.$, $\omega)$ ] and different contributions for the two-atom GaAs linear wire

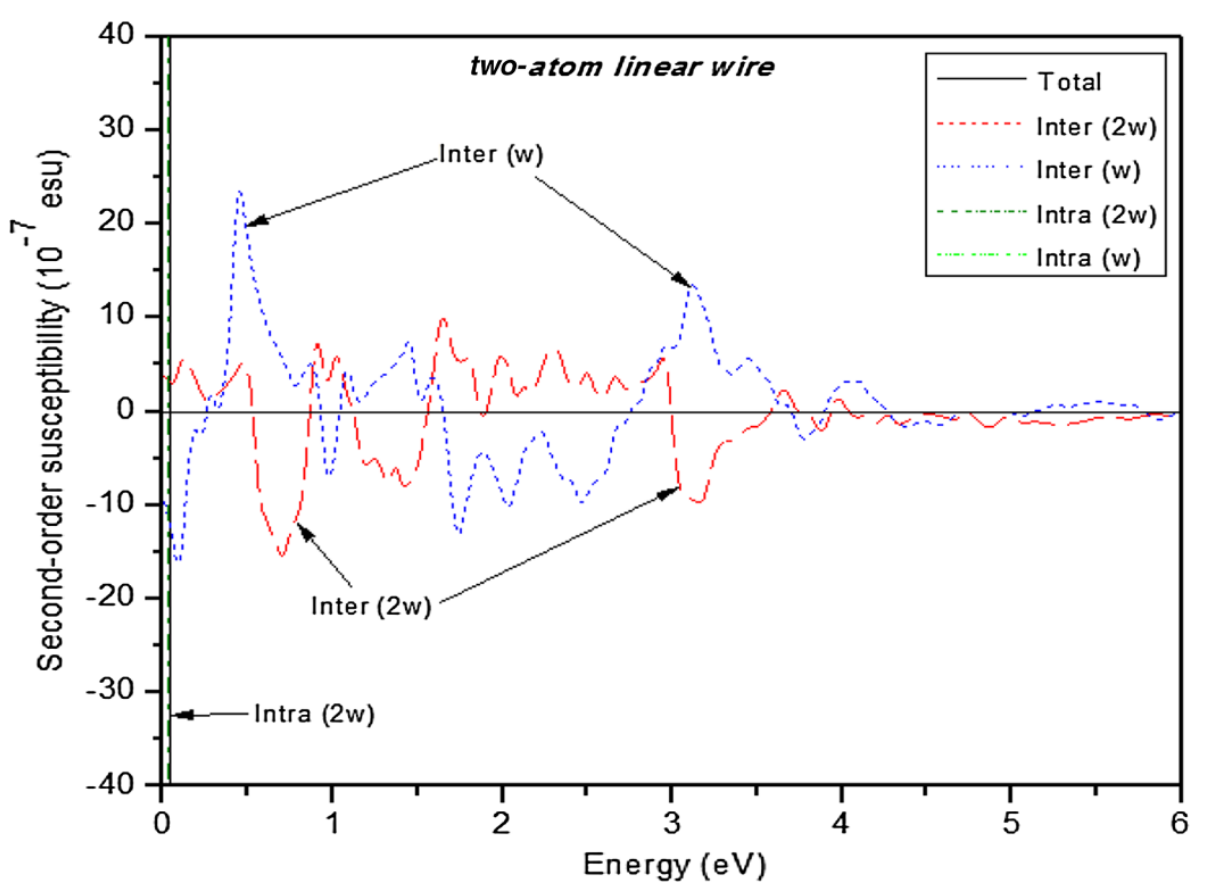

For the bulk spectra, the total SHG susceptibility is zero up to $3.5 \mathrm{eV}$ as depicted in Fig. 3, in the high-energy region, the SHG optical spectra are dominated by inter $(2 \omega)$ contribution towards the positive susceptibility and intra $(2 \omega)$ towards the negative region in the graph. For the two-atom linear wire (Fig. 4), the total SHG peaks are dominant near lower energy values, having exceptionally high susceptibility with intense peaks magnitude; here, the major contributions come from inter $(\omega)$ transition towards positive side and inter $(2 \omega)$ transitions towards the negative side. In case of two-atom zigzag wire (Fig. 5), the complex peaks are found intermingled with various contributions. It reveals that major SHG spectrum occurs between 0.5 and $6 \mathrm{eV}$ regions. We find peaks from inter $(2 \omega)$ transition in upper part and inter $(2 \omega)$ transition in lower part are obtained only two peaks near 0.5 and $1.5 \mathrm{eV}$ possesses inter $(2 \omega)$ transitions.

For four-atom square wire cross-section (Fig. 6), the total SHG as well as intra $(\omega)$ and intra $(2 \omega)$ transitions 
Fig. 5 Calculated second-order susceptibility $I_{m}\left[\chi_{z z z}(2 \omega, \omega\right.$, $\omega)]$ and different contributions for the two-atom GaAs zigzag wire

Fig. 6 Calculated second-order susceptibility $I_{m}\left[\chi_{z z z}(2 \omega, \omega\right.$, $\omega)$ ] and different contributions for the four-atom $\mathrm{GaAs}$ square wire
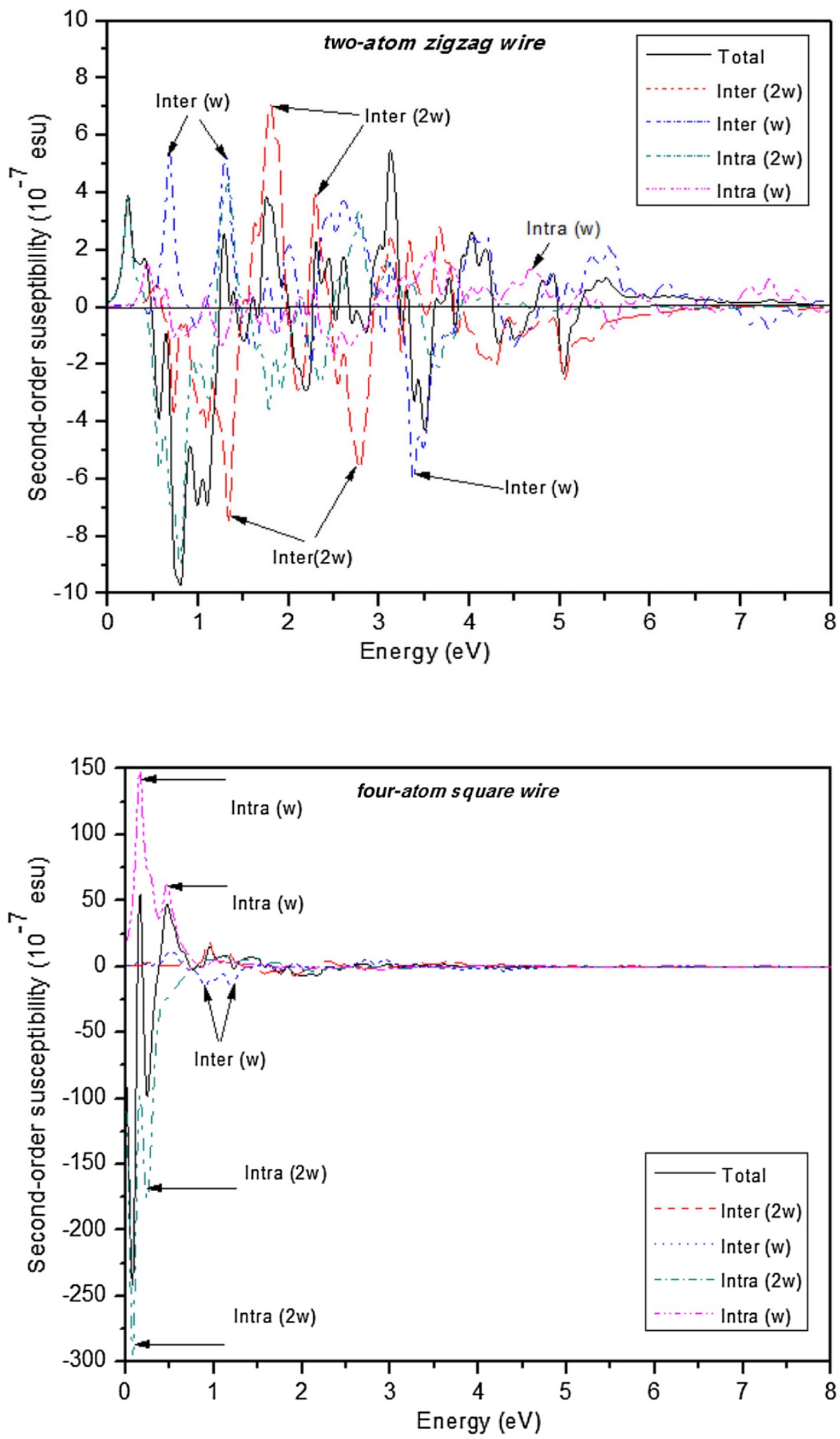

have greater contribution near lower energy region in contrast to higher energy side. The remarkable features account that the magnitude of SHG spectra is high in this type of configuration. In the positive SHG spectra, highest absorption spectra come from intra $(\omega)$ in the lower energy side, whereas in the negative SHG spectra the highest contribution comes from intra $(2 \omega)$ plus total transition.

Figure 7 shows spectra of six-atom hexagonal wire, the spectra include all the transitions, i.e., inter- and intracontribution. Many peaks of various magnitudes in 
Fig. 7 Calculated second-order susceptibility $I_{m}\left[\chi_{z z z}(2 \omega, \omega\right.$, $\omega)]$ and different contributions for the six-atom GaAs hexagonal wire

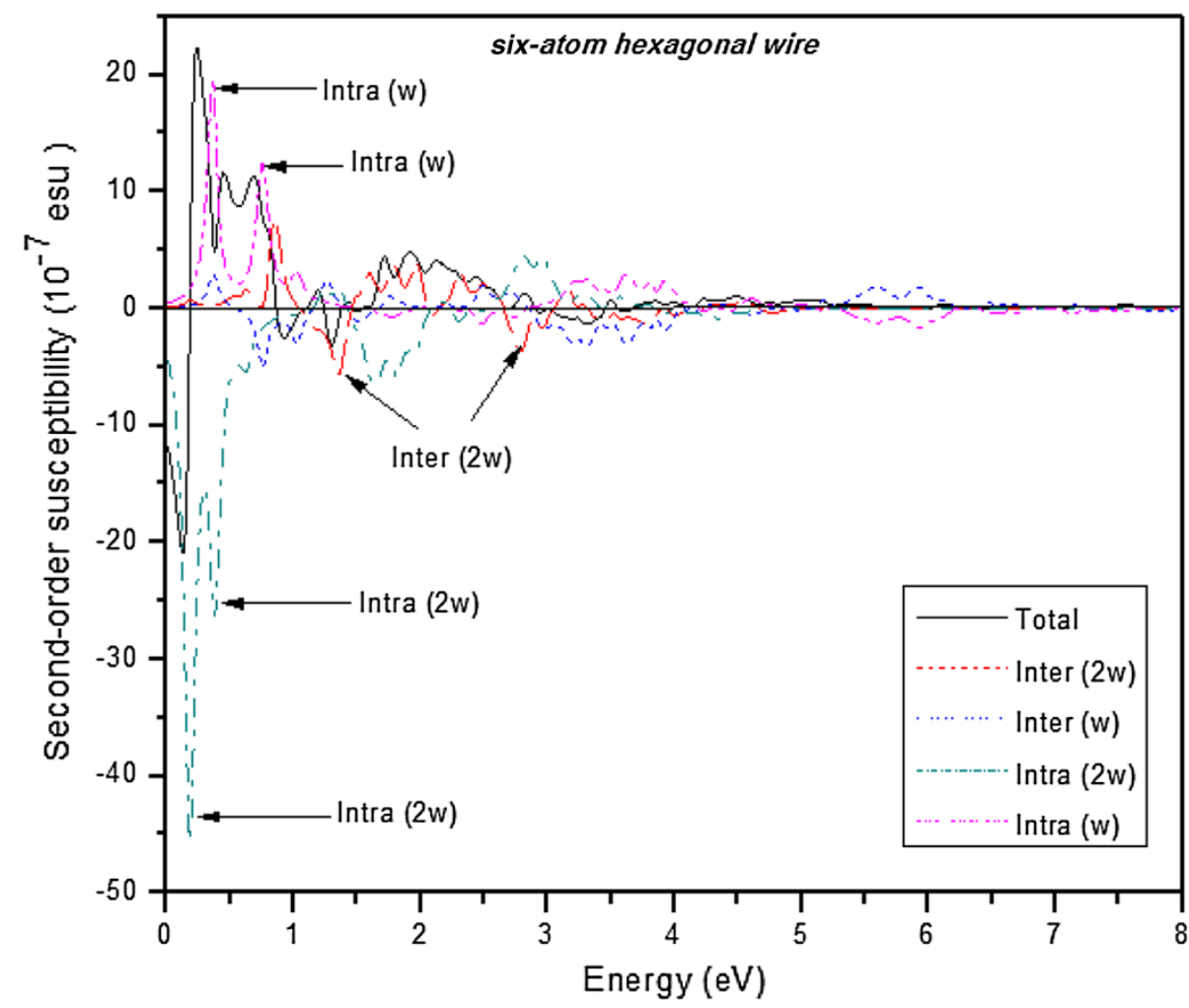

between 0.0 and $3.0 \mathrm{eV}$ region are observed, but the magnitudes are of low value in comparison to other configurations; thus, the highest absorption in the positive as well as negative spectra occurs due to intra $(\omega)$ and intra $(2 \omega)$ transitions, respectively.

\section{Conclusions}

We have calculated and analyzed the linear and nonlinear optical response of four different structures of GaAs nanowires. The aim of our analysis was to explore the best possible configuration of GaAs nanowire to be applied in photonic and optoelectronic devices. First, we have analyzed the linear response of the considered structures. The analysis of linear spectra reveals that strongest absorption occurs for four-atom square wire. In the stability analysis and electonic properties of GaAs nanowire, we found that the four atom square wire has the highest stability, thus it can be predicted that the structure that has the highest stability, must also have the strongest absorption. Second, we have investigated the SHG susceptibility spectra of these structures. In SHG spectra, we observe remarkable features for all structures. We predict that strongest absorption occurs for four-atom square nanowire configuration. We also revealed that the calculated peaks not only get sharper, but also show pronounced energy shift. This is mainly due to interband contribution to the imaginary part of the dielectric function. All SHG spectra comprise total, interand intra-band contributions. The SHG spectra are rather complicated due to various microscopic features observed. We have not come across any of the experimental or theoretical results to compare such type of linear and SHG optical spectra for various structures of GaAs nanowires. The present investigation is important, because size, shape and structure are the important criteria in nano-regime which one must not ignore so far as nanowires are concerned. Thus these types of semiconductor GaAs nanowires offer many opportunities for the assembly of nanoscale electronic and optoelectronic devices and applicable as interconnects.

Acknowledgments The authors are thankful to Department of Physics of Shri Ram College of Engineering and Management, Banmore, Morena (M.P.), and Computational Nanoscience and Technology Laboratory (CNTL) of ABV-Indian Institute of Information Technology and Management (ABV-IIITM), Gwalior (M.P.), for providing the infrastructural facilities for computational work.

Open Access This article is distributed under the terms of the Creative Commons Attribution License which permits any use, distribution, and reproduction in any medium, provided the original author(s) and the source are credited. 


\section{References}

Collins PG, Avouris P (2000) Nanotubes for electronics. Sci Am 283:62

Dekker C (1999) Carbon nanotubes as molecular quantum wires. Phys Today 52:22

Duan X, Wangand J, Lieber CM (2000) Synthesis and optical properties of gallium arsenide nanowires. Appl Phys Lett 76:1116

Duan X, Huang H, Cui Y, Wang J, Lieber CM (2001) Indium phosphide nanowires as building blocks for nanoscale electronic and optoelectronic devices. Nature 409:66

Gonze X, Beuken JM, Caracas R, Detraux F, Fuchs M, Rignanese GM, Sindic L, Verstraete M, Zerah G, Jollet F, Torrent M, Roy A, Mikami M, Ghosez P, Raty JY, Allan DC (2002) Firstprinciples computation of material properties: the ABINIT software project. Comput Math Sci 25:478

Hoang TB, Zhou H, Moses A, Dheeraj A, Helvoort A, Fimland BO, Weman H (2009) Observation of free exciton photoluminescence emission from single wurtzite GaAs nanowires. Appl Phys Lett 94:133105

Hohonberg P, Kohn W (1964) Inhomogeneous electron gas. Phys Rev 136:B864

Ihn SG, Song JI (2007) Morphology- and orientation-controlled gallium arsenide nanowires on silicon substrates. Nano Lett 7:39

Joyce HJ, Kim Y, Gao Q, Tan HH, Jagdish C (2006) Growth, structural and optical properties of GaAs/AlGaAs core/shell nanowires with and without quantum well shells, The Australian National University, Canberra, pp ACT0200

Joyce HJ, Gao Q, Tan HH, Jagdish C, Kim Y, Zhang X, Guo Y, Zou J (2007) Twin-free uniform epitaxial GaAs nanowires grown by a two-temperature process. Nano Lett 7:921

Khare SV, Gade V, Shi N, Ram Prasad R (2009) Effect of structure, surface passivation, and doping on the electronic and optical properties of GaAs nanowire: a first principles study. In: Bulletin of the American Physical Society 2009 APS March Meeting, vol 54:D26.0001

Kim Y, Gao Q, Joyce HJ, Tan HH, Jagdish C, Paladugu M, Zou J (2006) III-V nanowires for optoelectronics. Asia Pac Opt Commun 13:635226

Kohn W, Sham LJ (1965) Self-consistent equations including exchange and correlation effects. Phys Rev 140:A1133

Law M, Goldberger J, Yang Y (2004) Semiconductor nanowires and nanotubes. Ann Rev Mater Res 34:83
Lieber CM (2001) The incredible shrinking circuit. Sci Am 285:58

Lieber CM (2003) Nanoscale science and technology: building a big future from small things. MRS Bull 28:486

Martin RM (2009) Electronic structure. Cambridge University Press, Cambridge

Niquet YM (2006) Electronic and optical properties of InAs/GaAs nanowire superlattices. Phys Rev B 74:155304

Perdew JP, Burke K, Ernzerhof M (1996) Generalized gradient approximation made simple. Phys Rev Lett 77:3865

Redliski P, Peters FM (2008) Optical properties of free-standing GaAs semiconductor nanowires and their dependence on the growth direction. Phys Rev B 77:753329

Singh S, Srivastava P (2013) Optical properties of gallium phosphide $(\mathrm{GaP})$ nanowires. Appl Nanosci 3:89

Singh S, Srivastava P, Mishra A (2009a) Ab-initio study of gallium arsenide nanowires. J Comput Theor Nanosci 6:1556

Singh S, Srivastava P, Mishra A (2009b) Phys E 42:46

Srivastava P, Singh S (2008) Linear and second-order optical response of different GaN nanowires. Phys E 40:2742

Srivastava P, Singh S (2011) Stability analysis of AlN nanowire. J Comput Theor Nanosci 8:1764

Srivastava P, Singh S, Mishra A (2008) Stability and electronic properties of $\mathrm{GaN}$ nanowires: an ab initio approach. J Comput Theor Nanosci 5:635

Srivastava P, Singh S, Mishra A (2011) Electronic properties of GaP nanowires of different shapes. J Nanosci Nanotechnol 11:10464

Titova LV, Hoang TB, Jackson HE, Smith LM, Yarrison JM, Kim Y, Joyce HJ, Tan HH, Jagdish C (2006) Temperature dependence of photoluminescence from single core-shell GaAs-AlGaAs nanowires. Appl Phys Lett 89:173126

Troullier N, Martins JL (1991) Efficient pseudopotentials for planewave calculations. Phys Rev B 43:1993

Xia X, Yang P, Sun Y, Wu Y, Mayers B, Gates B, Yin Y, Kim F, Yan H (2003) One-dimensional nanostructures: synthesis, characterization, and applications. Adv Mater 5:353

Zhang G, Tateno K, Sanada H, Tawara T, Gotoh H, Nakano H (2009) Synthesis of GaAs nanowires with very small diameters and their optical properties with radial quantum-confinement effect. Appl Phys Lett 95:123104

Zou J, Paladugu M, Wang H, Auchterlonie GJ, Gao Y, Kim Y, Gao QH, Joyce HJ, Tan HH, Jagdish C (2007) Growth mechanism of truncated triangular III-V nanowires. Small 3:389 